\title{
Discovery of an unusual radio galaxy with a hybrid double-double and X-shaped morphology
}

\author{
C. C. Wang ${ }^{1,2} \dagger$, H. Yan. Zhou ${ }^{1,2,3}$, T. Ji $\mathbf{i}^{1,2,3}$, \\ W. J. Liu ${ }^{1,2}$ and N. Jiang ${ }^{1,2}$ \\ ${ }^{1}$ Key Laboratory for Research in Galaxies and Cosmology, University of Science and \\ Technology of China, Chinese Academy of Sciences, Hefei, Anhui 230026, China \\ ${ }^{2}$ Department of Astronomy, University of Science and Technology of China, \\ Hefei, Anhui 230026, China \\ ${ }^{3}$ Polar Research Institute of China, Jinqiao Rd. 451, Shanghai 200136, China
}

\begin{abstract}
We report the discovery of the first 'X'-shaped double-double radio galaxy (DDRG), MRC0929+164 (J0932+1611), from FIRST radio survey. The intersection angle of the lines of two pairs of radio lobes is around $20.6^{\circ}$ for this object, much larger than other DDRGs. This unusual morphology may be a hint that the growth of its central super-massive black hole suffered a violent galaxy merger once.
\end{abstract}

Keywords. Galaxies:jets, radio continuum:galaxies, black hole physics

\section{Introduction}

'Double-double radio galaxies' (DDRGs) constitute a special sub-class of Fanaroff-Riley type II radio galaxies (FR II). A typical DDRG contains two pairs of radio lobes with a common center, which can be regarded as strong evidence of episodic activity of the central engine. There are $\sim 12-17$ such known objects up to date (see Brocksopp et al. (2011) and references therein). The multiple lobes in these radio galaxies, however, are well aligned with each other within a few degrees. The overall linear radio morphology of the known DDRGs suggests that global dynamics of jet ejection from the central engine remains rather stable between two active epochs.

Our research interests then focus on searching the VLA FIRST radio survey to find possible DDRG candidates which do not reveal classical linear radio morphology. Such unusual DDRGs may provide new information on the dynamics of the system.

\section{Data Analysis and Results}

We write a simple IDL code to read the downloaded FIRST cutout image files (in FITS format). By visual inspection, we attempt to mark the possible lobe areas interactively and save the results into a parameter file. With great efforts, we finally discovery an unusual radio galaxy MRC0929+164 (or J0932+1611). Fig. (a) gives the contour map of the this radio galaxy (green line) extracted from FIRST radio survey, overlaid with the matching SDSS image of the central unresolved nucleus. The flux-weighted centers of two pairs of lobes are calculated and linked together. Then we measure the intersection angle to be $20.6^{\circ}$, much larger than other known DDRGs. Fig. (b) presents best-fit of the continuum and emission lines of the optical nucleus of MRC0929+164. The derived

\section{$\dagger$ Correspondence author: ccwang@ustc.edu.cn}




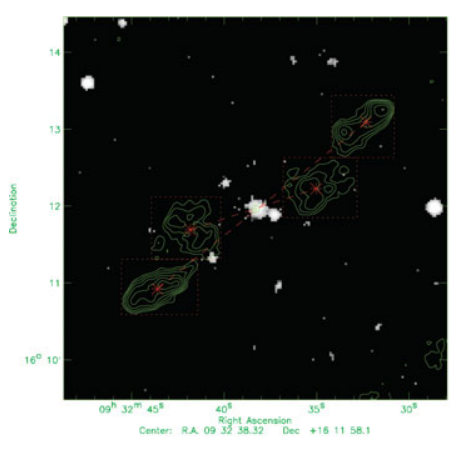

(a)

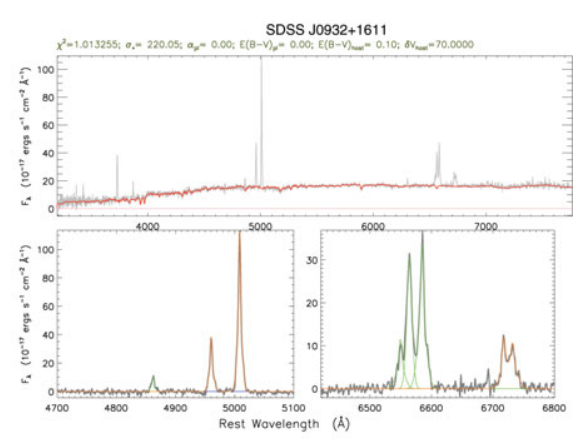

(b)

Figure 1. (a) Contour map of the radio galaxy MRC0929+164 (green line) extracted from FIRST radio survey, overlaid with the matching SDSS image of the central unresolved nucleus; (b) Best-fit of the continuum and emission lines of the optical nucleus of MRC0929+164. The top panel indicates the overall spectrum, and two bottom panels are zoom-in of the local spectrum.

emission line redshift of the object is $z=0.191$. The best fit of the optical continuum $\left(\chi^{2}=1.01\right)$ is based upon BC03 template of simple stellar population (SSP) analysis (Bruzual \& Charlot (2003)). The SSP age of the galaxy is around 2.75Gyr. The fit also gives the stellar velocity dispersion of the galaxy, i.e. $\sigma_{*}=220.05 \mathrm{~km} / \mathrm{s}$. With the $M_{\mathrm{BH}}-\sigma_{*}$ relation of Gültekin et al. (2009), the mass of central super-massive black hole is estimated to be $(2.0-2.5) \times 10^{8} M_{\odot}$.

\section{Conclusion and Discussion}

We discover the first ' $\mathrm{X}$ ' shaped morphology DDRG. The outer lobes of MRC0929+164 are more compact, which implies they suffer less dissipation and form later than the inner lobes. Considering the 3D orientation of the lobes, the related jets (as well as the black hole-accretion disk system) have changed direction seriously. This can be treated as evidence that the growth history of the central massive black hole may suffer a violent galaxy merger.

\section{Acknowledgement}

This work is supported by the Fundamental Research Funds for the Chinese Central Universities with grants WK2030220006 and WK2030220004. We also acknowlege the support from Chinese Natural Science Foudation through grants NSFC (11033007, 10973012, 11073019) and 973 program (2007CB815405).

\section{References}

Brocksopp, C., Kaiser, C. R., Schoenmakers, A. P., \& de Bruyn, A. G. 2011, MNRAS, 410, 484 Bruzual, G. \& Charlot, S. 2003, MNRAS, 344, 1000

Gültekin, K., et al. 2009, ApJ, 698, 198 